\title{
An Investigation of the Small Eccentricity in the Spectroscopic Binary System $\zeta \operatorname{Tr} A$
}

\author{
S. Komonjinda, J.B. Hearnshaw, and D.J. Ramm
}

Department of Physics and Astronomy, University of Canterbury, Christchurch, New Zealand email: sko18@student.canterbury.ac.nz

\begin{abstract}
.
The orbital eccentricity of the SB1 system $\zeta$ TrA (S.T. F9V, $P \sim 13 \mathrm{~d}$ ) was found by Skuljan et al. (2004) to be $e=0.01398 \pm 0.00019$. Lucy (2005) devised a statistical test of the significance of this result, based on the amplitude and phase of the third harmonic in the Fourier analysis of the radial velocity data, and concluded that the non-zero eccentricity measured does not arise from a slightly eccentric Keplerian orbit, but from proximity effects in the binary. He therefore believes a circular orbit should be assigned to this system. In this paper we investigate one possible proximity effect, namely the tidal distortion of the primary star, such that the measured Doppler shift does not accurately indicate the centre of mass radial velocity of the star as a whole. The code of Wilson \& Devinney (2003) was used to model the tidal distortion of the measured radial velocities, assuming a range of possible secondary masses, corresponding to M-dwarf companions. The result is that even for the lowest possible mass secondary of $0.09 M_{\odot}$ with $\sin i=1$ (this gives the greatest tidal distortion, as it is closest to the primary) there is no significant effect on the radial velocities (the differences are of order $1 \mathrm{~m} \mathrm{~s}^{-1}$ as a result of the tidal effects). Similar negligible tidal effects arise using a white dwarf companion. We note that the difference between a circular orbit and the observations amounts to as much as $140 \mathrm{~m} \mathrm{~s}^{-1}$ at some phases, which is essentially the amplitude of the second harmonic in the data. Our conclusion is that this strong and highly significant second harmonic is most probably the result of a small orbital eccentricity as reported by Skuljan et al. (2004). We note that the observed third harmonic according to Lucy (2005) has an amplitude of only $5.2 \pm 2.0 \mathrm{~m} \mathrm{~s}^{-1}$, which is just over twice the error bar of its measurement, and that the predicted third harmonic for an eccentric orbit is only $1.6 \mathrm{~m} \mathrm{~s}^{-1}$.
\end{abstract}

Keywords. binaries: close, binaries: spectroscopic

\section{Introduction}

In a recent paper, Skuljan et al. (2004) derived precise elements of a Keplerian orbit for the $\mathrm{F} 9 \mathrm{~V}$ star $\zeta \operatorname{TrA}(\mathrm{V}=4.91)$ in a single-line spectroscopic binary. They used 228 high-precision radial velocities from a high-resolution fibre-fed echelle spectrograph, Hercules. The results indicated a nearly circular orbit with small but highly significant eccentricity $(e=0.01398 \pm 0.00019)$. Subsequently Lucy (2005) criticized this orbital solution on the basis that the weak third harmonic in the radial-velocity power spectrum was slightly stronger than the third harmonic expected from a Keplerian orbit and with the wrong phase. He instead advocated a perfectly circular orbital solution for this star, consistent with the recommendations of Lucy \& Sweeney (1979), and he ascribed the highly significant departures of the measured velocities (by up to 10 $\sigma$ ) to proximity effects of unspecified type. In this paper we investigate the plausibility that any such 'proximity effects' might give a spurious eccentricity for $\zeta \operatorname{TrA}$. We study the tidal distortion of the primary star, the reflection effect and the effect of starspots on the radial-velocity solution. Henry et al. (1996) note that $\zeta \operatorname{TrA}$ is an active star from the $H$ and $K$ flux. 
Table 1. Derived orbital parameters of $\zeta$ TrA, from Skuljan et al. (2004)

\begin{tabular}{cl}
\hline Parameter & Value \\
\hline$K_{1}$ & $7.5001 \pm 0.0017 \mathrm{~km} \mathrm{~s}^{-1}$ \\
$e$ & $0.01398 \pm 0.00019$ \\
$\omega_{1}$ & $252^{\circ} .30 \pm 0^{\circ} .87$ \\
$T_{0}$ & HJD $2452761.413 \pm 0.031$ \\
$P$ & $12.975780 \mathrm{~d}$ (fixed) \\
$V_{0, \text { rel }}$ & $6.3892 \pm 0.0012 \mathrm{~km} \mathrm{~s}^{-1}$ \\
& (not barycentric corrected) \\
\hline
\end{tabular}

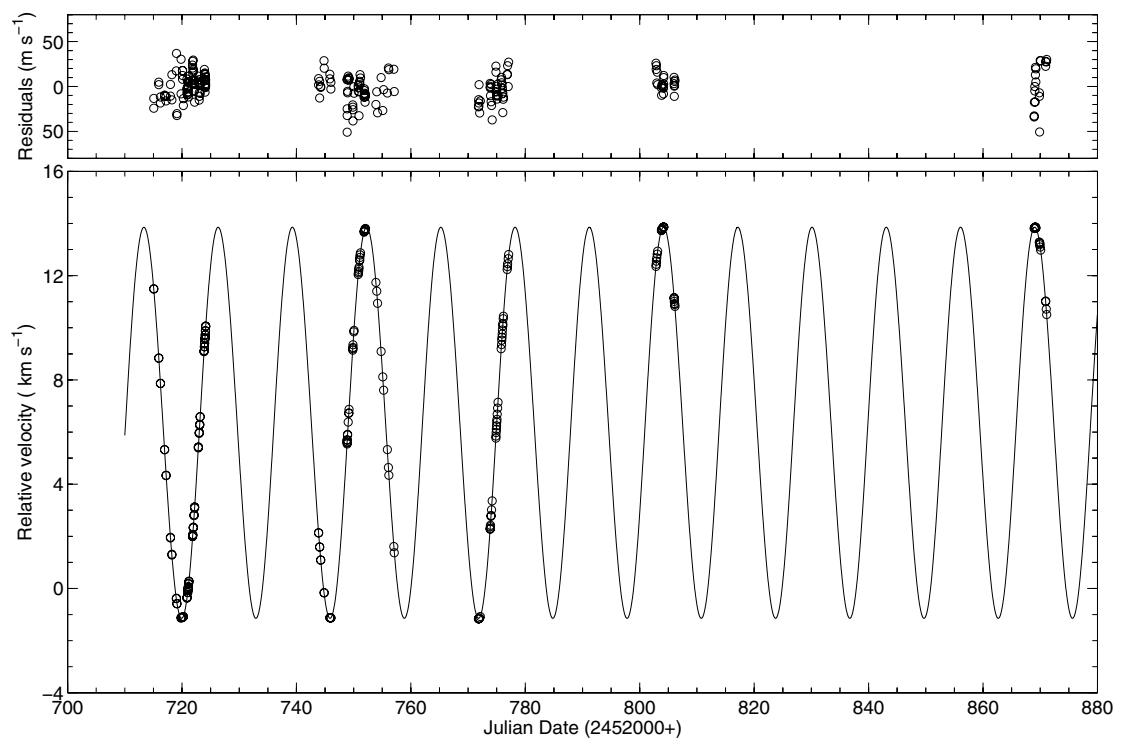

Figure 1. The radial velocity curve of $\zeta$ TrA observed at Mt. John University Observatory, New Zealand from Skuljan et al. (2004) and the residual velocities when fitting the data with a Keplerian elliptical orbit. The root mean squared error of the fit is $14 \mathrm{~m} \mathrm{~s}^{-1}$.

However our recent spectra (2006) show negligible chromospheric emission. King et al. (2003) assign $\zeta$ TrA to the UMa moving group and it must therefore be young $(\sim 500$ Myr).

Lucy (2005) notes that the third harmonic of a Keplerian orbit is $9 / 8 K e^{2}$ which for $\zeta$ $\operatorname{TrA}$ is $1.65 \mathrm{~m} \mathrm{~s}^{-1}$. However the observed third harmonic is $5.2 \pm 2.0 \mathrm{~m} \mathrm{~s}^{-1}$, and this has a phase some $90^{\circ}$ different from the expected value. He estimates that the probability of this occurring by chance is less than $1 \%$. As pointed out by Lucy, the Keplerian third harmonic should be undetectable with the precision of the data $\left(\sigma \sim 14 \mathrm{~ms}^{-1}\right.$ for one data point). Lucy instead prefers to ascribe a circular orbit to $\zeta \operatorname{Tr} A$ and all the second and higher harmonics are then attributed to 'proximity effects'. 


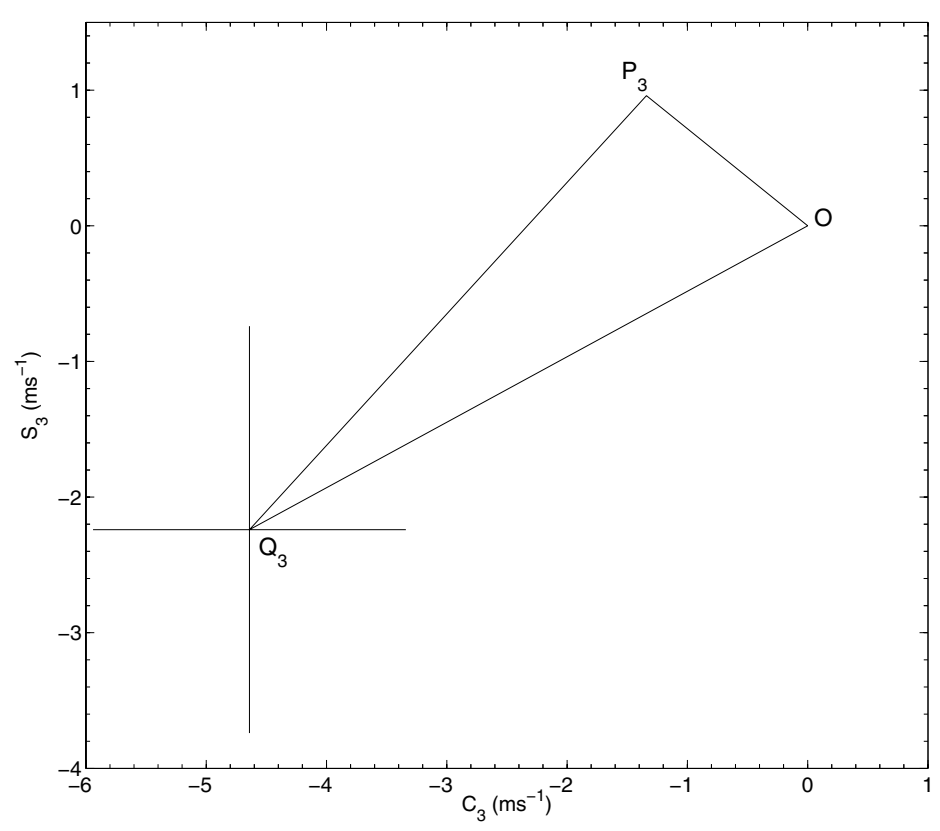

Figure 2. The $3^{\text {rd }}$ harmonic components in the velocity curve of $\zeta$ TrA in a phasor diagram according to Lucy (2005). OP3 is the expected Keplerian $3^{\text {rd }}$ harmonic, while $O Q 3$ is the observed $3^{\text {rd }}$ harmonic with its error bars.

\section{Theoretical models of $\zeta \operatorname{Tr} \mathrm{A}$ with tidal distortion and the reflection effect}

We generated a model of $\zeta$ TrA from the 2003 version of the Wilson-Devinney (1971) computer program in order to match the observational data from Skuljan et al. (2004). The model included both tidal distortion and the reflection effect, both of which in principle cause small perturbations of the radial velocity from the true centre of mass value for the primary star. The secondary star was assumed to be between M1V and $\mathrm{M} 7 \mathrm{~V}$ as there are maximum and minimum limits from the mass function and light ratio (see Skuljan et al. 2004). The RV curves generated from the program for three models $(\mathrm{F} 9 \mathrm{~V}+\mathrm{M} 1 \mathrm{~V}$ without proximity effects, F9V+M1V with proximity effects and F9V+M7V with proximity effects), using the orbital parameters as derived by Skuljan et al. (2004), show no difference in radial velocities between the models and observational data. What is more the models with either type of proximity effect differ from those for circular orbits and no proximity effects by less than $1 \mathrm{~m} \mathrm{~s}^{-1}$. If such small perturbations were to be added to the data from a truly circular orbit, the measured eccentricity would still be $e=0.000$. The effect of tidal surface distortion for $\zeta$ TrA as calculated by Budding (2006) is around $0.75 \mathrm{~cm} \mathrm{~s}^{-1}$.

The models of circular orbit show no evidence for proximity effects from tidal distortion and reflection effects that can cause the spurious eccentricity of 0.01398 . The difference between a circular orbit and the observations amounts to as much as $140 \mathrm{~m} \mathrm{~s}^{-1}$ at some phases, which is essentially the amplitude of the second harmonic in the data. 
Table 2. Parameters for spot model calculations

\begin{tabular}{ccccccc}
\hline Model & $N_{\text {spots }}$ & $\begin{array}{c}A_{\text {spot }} \\
(\%)\end{array}$ & $\begin{array}{c}\ell \\
(\circ)\end{array}$ & $\begin{array}{c}\text { fictitious } \\
e\end{array}$ & $\begin{array}{c}3^{\text {rd }} \text { harmonic } \\
\left(\mathrm{m} \mathrm{s}^{-1}\right)\end{array}$ & $\begin{array}{c}\Delta V \\
\text { (semi-amplitude magnitude) }\end{array}$ \\
\hline$\# 1$ & 1 & 12 & - & 0.0101 & 44.1 & 0.047 \\
$\# 2$ & 2 & 8,8 & 90,270 & 0.0147 & 5.0 & 0.032 \\
$\# 3$ & 3 & $8,7,3$ & $108,270,280$ & 0.0134 & 21.5 & 0.034 \\
\hline
\end{tabular}

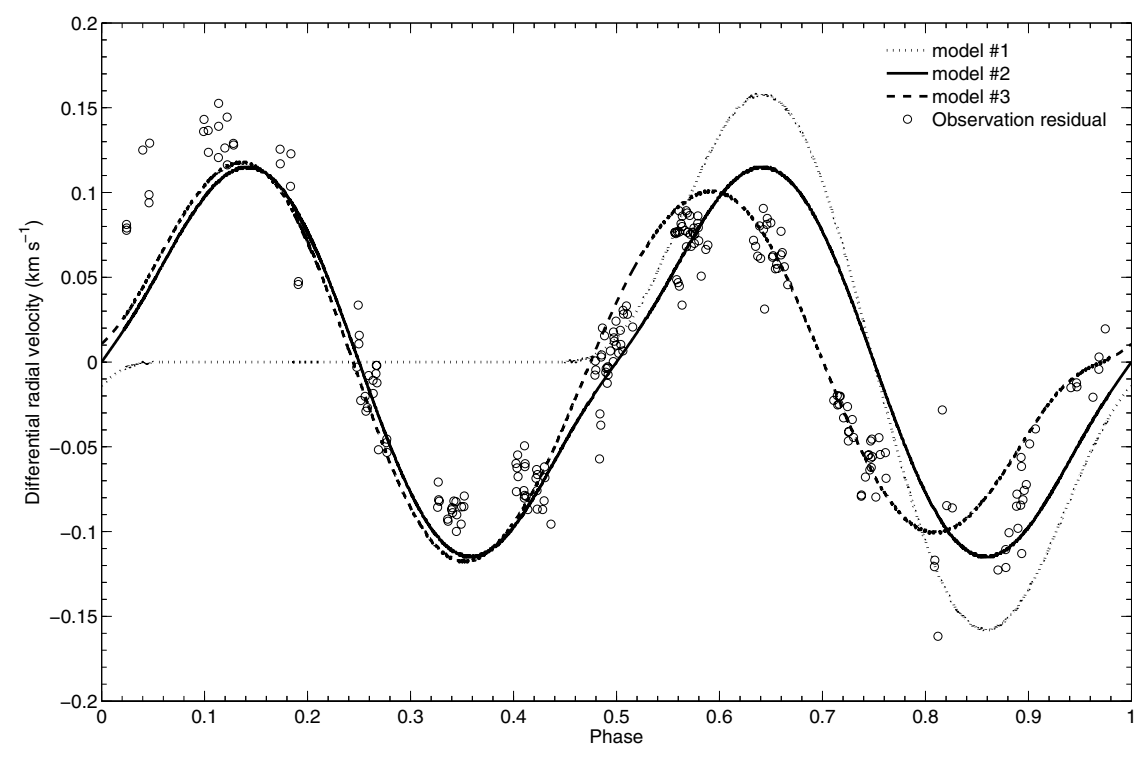

Figure 3. The difference in the radial velocities computed from Wilson-Devinney code between each spot model and a model with no spot. The blue dash line is from a model with a $12 \%$ area spot. The green line is from a model with two $8 \%$ spots and a red dash-dot line is from a model with three spots at $8 \%, 7 \%$ and $3 \%$ area. All models have circular orbits.

\section{Spot models for $\zeta \operatorname{Tr} A$}

We have used the Wilson-Devinney code also to investigate the effect of spots on the radial-velocity curve, as proposed by Sterne (1941) and Hatzes (2002). Our models with spots $\left(\Delta T=10^{3} \mathrm{~K}\right)$ assumed a strictly circular orbit with co-rotating stars and equatorial spots, and they were used to compute theoretical radial velocities which were then analysed using our Keplerian orbit analysis code to obtain fictitious orbital elements.

Table 2 shows three models with respectively one, two and three spots of area $A_{\text {spot }}$ as a percentage of the projected hemisphere, and longitude such that $\ell=0$ corresponds to the line between the star centres. Figure 3 shows the difference in the radial velocities from the models and those from a circular orbit and no spots. The maximum observed difference from the real data is $140 \mathrm{~ms}^{-1}$, and the spot models investigated can give velocity perturbations of that order. Moreover the fictitious eccentricities obtained by assuming Keplerian orbits and no spots were of the order of 0.01 for these three models, comparable to the observed value of $\mathrm{e}=0.013938$. We found that the third harmonic was very sensitive to the spot longitudes. However model \#2 above has a third harmonic amplitude of $5.0 \mathrm{~m} \mathrm{~s}^{-1}$, similar to the value observed.

All spot models should result in a photometric spot wave variability of semi-amplitude 
$\Delta \mathrm{V}$. Hipparcos photometry shows constancy of light to 0.006 magnitude, quite a lot less than predicted from the models, but more than the photometric precision. However Hipparcos observations were a decade earlier than our velocity data, and the spots may have been less then. A period analysis of 238 Hipparcos photometric data points gave no detectable period near the 12.9-day orbital period.

\section{Conclusions}

The SB1 system $\zeta$ TrA shows a small orbital eccentricity when the velocities are fitted to a Keplerian orbit. This cannot be explained by proximity effects such as tidal distortion or the reflection effect. However, starspots can result in fictitious eccentricity values and may also reproduce a weak non-Keplerian third harmonic, similar to that found by Lucy (2005). This does not prove that $\zeta$ TrA has spots.

\section{Acknowledgements}

S.K. would like to express her thanks to the IAU and the Royal Thai Government for the grant that enabled her to attend this conference. The work of S.K. at the University of Canterbury is supported by a Royal Thai Government Scholarship.

\section{References}

Budding E. 2006, (Private communication)

Henry, T.J., Soderblom, D.R., Donahue, R.A., \& Baliunas, S.L. 1996, AJ 111, 439

Hilditch, R.W. 2001, An Introduction to Close Binary Stars, Cambridge University Press, Cambridge

King, J.R., Villarreal, A.R., Soderblom, D.R., Gulliver, A.F., \& Adelman, S.J. 2003, AJ 125, 1980

Lucy, L.B. \& Sweeney, M.A. 1971, AJ 76, 544

Lucy, L.B. 2005, A\&SA 439, 663

Skuljan, J., Ramm, D.J., \& Hearnshaw, J.B. 2004, MNRAS 325, 975

Sterne, T.E. 1941, Proc Nat Acad Sci 27, 168

Wilson, R.E. \& Devinney, E.J. 1971, ApJ, 166, 605 